Proceedings

\title{
Developing an Einsteinian curriculum for school science ${ }^{\dagger}$
}

Tejinder Kaur

Publisher's Note: MDPI stays neutral with regard to jurisdictional claims in published maps and institutional affiliations.

\section{cc) (i)}

Copyright: (c) 2021 by the authors. Submitted for possible open access publication under the terms and conditions of the Creative Commons Attribution (CC BY) license (http://creativecommons.org/licenses /by/4.0/).

\begin{abstract}
The concept of curved space, warped time, gravity as a curvature of spacetime, light as photons, heat as phonons etc., are not a part of the Australian school curriculum or many other parts of the world. These concepts are often ignored as many consider them highly mathematical or only for elite students.

Despite being modern, these concepts have relevance in daily lives. People carry smartphones with them all the time and the working of the Global Positioning System (GPS) depends on the Einsteinian concepts. Apart from this, these concepts have tremendous applications, for example, computer chips, solar panels, medical imaging, and quantum computer.

An international project called the Einstein-First is developing the Einsteinian curriculum for years 3-10 Australian students. The project was founded on the premise that every child has the right to share our best understanding of physical reality. The team carefully reviewed the existing science curriculum and found many places where Einsteinian physics can be integrated. The team is developing a spiral curriculum. The concepts learnt at primary school curriculum are revisited in secondary school curriculum. For example, Year 3 students will learn about phonons, atoms and molecules while learning the concept of Heat. Year 5 students will learn about photons while learning the concept of light. The concept of light will then be revisited in Year 9, where students will learn it at a more advanced level. Similarly, Year 7 students will learn the concept of gravity in Einsteinian context and Year 8 students will learn the concept of energy. The team developed several roleplays where students will appreciate how scientists struggle over the years to understand the concept of light, black holes etc.

To support teachers, the team is preparing a detailed lesson plans along with teacher background documents and activity videos.The resources have been used in the Playing with Protons events for teachers at CERN, and have been translated in Greek and Italian.
\end{abstract}

Keywords: Einsteinian physics, primary school, secondary school 